\title{
Predictive markers for severe hypocalcemia in dialysis patients with secondary hyperparathyroidism after near-total parathyroidectomy
}

\author{
Yang Zou ${ }^{1 \#}$, Nianrong Zhang ${ }^{2 \#}$, Yun Tang ${ }^{1}$, Zhipeng Zhan ${ }^{3}$, Meng Yang ${ }^{4}$, Yao Lu $^{4}$, Gui-Sen Li ${ }^{1}$, Ling Zhang ${ }^{2}$ \\ ${ }^{1}$ Department of Nephrology, School of Medicine, Sichuan Academy of Medical Science and Sichuan Provincial People's Hospital, Sichuan Clinical \\ Research Center for Kidney Diseases, University of Electronic Science and Technology of China, Chengdu, China; ${ }^{2}$ Department of Nephrology, \\ China-Japan Friendship Hospital, Beijing, China; ${ }^{3}$ Department of Nephrology, Suining Central Hospital, Suining, China; ${ }^{4}$ Department of Breast and \\ Thyroid Surgery, China-Japan Friendship Hospital, Beijing, China \\ Contributions: (I) Conception and design: Y Zou, N Zhang; (II) Administrative support: GS Li, L Zhang; (III) Provision of study materials or patients: \\ M Yang, Y Lu; (IV) Collection and assembly of data: Y Tang; (V) Data analysis and interpretation: Z Zhan; (VI) Manuscript writing: All authors; (VII) \\ Final approval of manuscript: All authors. \\ "These authors contributed equally to this work and should be considered as co-first authors. \\ Correspondence to: Ling Zhang. Department of Nephrology, China-Japan Friendship Hospital, No. 2 Yinghua East Street, Chaoyang District, Beijing \\ 100029, China. Email: zhangling5@medmail.com.cn; Gui-Sen Li. Department of Nephrology, School of Medicine, Sichuan Academy of Medical \\ Science and Sichuan Provincial People's Hospital, Sichuan Clinical Research Center for Kidney Diseases, University of Electronic Science and \\ Technology of China, West 2nd Duan, 1st Circle Rd, Qingyang District, Chengdu 610072, China. Email: guisenli@163.com.
}

Background: Secondary hyperparathyroidism (SHPT) is common in dialysis patients with end-stage renal disease (ESRD). Parathyroidectomy (PTX) is an effective treatment for SHPT. Postoperative severe hypocalcemia (SH) is a common and severe complication after PTX. This study aimed to investigate the potential predictive markers of SH in dialysis ESRD patients with SHPT after near-total PTX (near-tPTX) without autotransplantation (AT).

Methods: A retrospective analysis involving 131 dialysis patients with SHPT who were treated with neartPTX without AT between January and August 2018 was performed. Demographic characteristics (age, gender, type of dialysis modality, etc.) and perioperative laboratory parameters [serum calcium, phosphorus, alkaline phosphatase (ALP), intact parathyroid hormone (iPTH), and bone metabolism markers] were collected and analyzed. Postoperative serum calcium level $<1.875 \mathrm{mmol} / \mathrm{L}(7.5 \mathrm{mg} / \mathrm{dL})$ was defined as postoperative $\mathrm{SH}$.

Results: Among the 131 patients, 73 (55.7\%) had postoperative hypocalcemia and $43(32.8 \%)$ had postoperative SH. Univariate analysis showed that values of preoperative serum iPTH, calcium, ALP, bonespecific alkaline phosphatase (BAP), and osteocalcin (OC) were significantly different between the SH and non-SH groups. In the multivariate logistic regression model, preoperative serum ALP was an independent risk predictor of postoperative $\mathrm{SH}$. The receiver operating characteristic (ROC) curve for preoperative serum ALP was 277 U/L. The sensitivity of preoperative serum ALP was $73.8 \%$ and the specificity was $63.2 \%$.

Conclusions: The incidence rates of postoperative hypocalcemia and SH in dialysis patients with SHPT after near-tPTX without AT were $55.7 \%$ and $32.8 \%$, respectively. Preoperative serum ALP was an independent predictor for the occurrence of postoperative SH, and dialysis patients with SHPT were susceptible to postoperative SH when preoperative serum ALP level was >277 U/L. Hence, we recommend that preoperative serum ALP be utilized to complement clinical protocols for postoperative SH management of dialysis ESRD patients with SHPT after near-tPTX without AT.

Keywords: Severe hypocalcemia (SH); secondary hyperparathyroidism (SHPT); dialysis; near-total parathyroidectomy (near-tPTX); alkaline phosphatase (ALP) 
Submitted Aug 11, 2021. Accepted for publication Sep 24, 2021.

doi: 10.21037/apm-21-2509

View this article at: https://dx.doi.org/10.21037/apm-21-2509

\section{Introduction}

Secondary hyperparathyroidism (SHPT) is a common complication of chronic kidney disease (CKD). The prevalence of SHPT increases with the progression of CKD (1-3), Which is $47.6 \%, 56.8 \%, 80.4 \%$ in CKD stage 3, 4, 5 patients (4). And develops in almost all patients with endstage renal disease (ESRD) (5). As metabolic disorder of calcium $(\mathrm{Ca})$, phosphorus $(\mathrm{P})$, and vitamin $\mathrm{D}$ are common in CKD patients, especially in ESRD patients with loss of renal function. Therefore, PTH which is a physiological hormone of regulating mineral balance (calcium, phosphorus and vitamin D) will be out of control and lead to hyperparathyroidism due to persistence of parathyroid gland cell proliferation and PTH hypersecretion (6). SHPT can cause high turnover bone disease [in which circumstance bone formation markers representing osteoblast activity including alkaline phosphatase (ALP) and so on will rise], bone fracture, cardiovascular disease, skin pruritus, anemia, myopathy, malnutrition, and neurological symptoms. SHPT can also seriously affect the quality of life of dialysis patients and is associated with all-cause mortality and cardiovascular events in dialysis patients (7-9). Treatments for mild and moderate SHPT include a limited-phosphorus diet, phosphorus binding agents, calcium-sensitive receptor agonists, and active vitamin analogs (10). For patients with refractory SHPT, parathyroidectomy (PTX) is recommended by the Kidney Disease: Improving Global Outcomes (KDIGO) guideline, published in 2017 (11). Previous studies have reported that PTX was necessary for about $15 \%$ of patients after 10 years of dialysis and $38 \%$ of patients after 20 years (12).

PTX can decrease PTH and quickly and effectively correct disturbed mineral homeostasis caused by SHPT. Thus, it improves quality of life, decreases the risk of bone fracture, and helps to improve malnutrition, insomnia, restless leg syndrome, depression, and anemia (13-16). PTX surgical approaches include total PTX with autotransplantation (tPTX + AT), subtotal PTX (sPTX), total PTX without autotransplantation (tPTX), and a modified version of sPTX called near-total PTX (near-tPTX), which retains fewer parathyroid glands than sPTX (6). The advantages of neartPTX are lower recurrence rates compared with sPTX or tPTX + AT, and less frequent occurrence of refractory hypocalcemia caused by tPTX, which requires calcium supplementation and calcitriol for a longer period (17).

Postoperative hypocalcemia is the most common complication following PTX. It was first reported in 1948 in patients with prolonged hypocalcemia after PTX for primary hyperparathyroidism (HPT) (18). The incidence of post-PTX hypocalcemia in SHPT patients varies from 20-85\% (17). Mild clinical symptoms include skeletal muscle cramps, weakness, ileus, headaches, paresthesia, and malabsorption. Severe hypocalcemia $(\mathrm{SH})$ is commonly accompanied by life-threatening sequelae such as respiratory muscle weakness, laryngeal stridor, seizures, cardiac arrhythmias, congestive heart failure, tetany, and possible sudden death (10). Therefore, early identification of the risk factors of SH after PTX can help to avoid serious consequences. However, the conclusions of existed papers referred to the risk factors of SH after PTX studies are controversial.

In this study, we aimed to investigate the potential predictive markers of $\mathrm{SH}$ and their diagnostic value in dialysis ESRD patients with SHPT after near-tPTX without AT. We present the following article in accordance with the STARD reporting checklist (available at https:// dx.doi.org/10.21037/apm-21-2509).

\section{Methods}

\section{Patients}

A retrospective cohort study was designed and performed. Consecutive dialysis patients with SHPT who received near-tPTX without AT at the China-Japan Friendship Hospital between January and August 2018 were recruited for the study. The inclusion criteria were (I) dialysis patients with SHPT, (II) patients treated with near-tPTX without AT, and (III) patients with detailed medical records. The exclusion criteria were patients with relapses and recurrences. The following data from medical records were retrospectively recorded: age, gender, type of dialysis modality, dialysis duration, height, body weight, smoking history, calcium supplementation, active vitamin D sterols, calcimimetics, preoperative/postoperative parameters 
[hemoglobin $(\mathrm{Hb})$, blood glucose (Glu), serum calcium (Ca), phosphorus (P), intact parathyroid hormone (iPTH), alanine transaminase (ALT), aspartate aminotransferase (AST)], and bone turnover markers [alkaline phosphatase (ALP), bone alkaline phosphatase (BAP), osteocalcin (OC), type I procollagen amino-terminal propeptide (PINP), $\beta$-cross-linked C-terminal telopeptide of type I collagen $(\beta-\mathrm{CTX})$, tartrate-resistant acid phosphatase (TRAcP)]. The laboratory data was obtained before surgery and 0-24 hours after PTX. The study was approved by the Hospital Human Research Ethics Committee of China-Japan Friendship Hospital (No. 2019-SDZL-12). Given the retrospective nature of the study, formal informed consent was exempted by our institutional board. The study was conducted in accordance with the guidelines of the Declaration of Helsinki (as revised in 2013).

\section{Surgical indications}

According to KDIGO guidelines (19) and previous literature (20), the surgical indications of PTX include: (I) iPTH $>800 \mathrm{pg} / \mathrm{mL}$ (severe SHPT), with hypercalcemia or hyperphosphatemia; (II) clinical symptoms of SHPT refractory to medical treatment, including severe bone ache, skin pruritus, external calcification, and bone deformity; (III) drug resistance; and (IV) imaging examination, including neck ultrasonography and parathyroid scintigraphy with technetium-99m methoxyisobutylisonitrile, identifying at least 1 enlarged parathyroid gland. Surgery was considered in patients who conformed to all the above criteria. Near-tPTX surgery was performed in all enrolled patients.

\section{Definition of hypocalcemia and SH}

A total serum calcium level $<2.10 \mathrm{mmol} / \mathrm{L}(8.4 \mathrm{mg} / \mathrm{dL})$ after PTX was defined as postoperative hypocalcemia. A serum calcium level $<1.875 \mathrm{mmol} / \mathrm{L}(7.5 \mathrm{mg} / \mathrm{dL})$ after PTX was defined as postoperative SH. A cutoff of $1.875 \mathrm{mmol} / \mathrm{L}$ was chosen because symptoms of hypocalcemia develop when serum $\mathrm{Ca}<1.875 \mathrm{mmol} / \mathrm{L}(21)$.

\section{Laboratory assays}

Laboratory parameters were routinely measured in all $(\mathrm{n}=131)$ patients before surgery, including $\mathrm{Ca}, \mathrm{P}, \mathrm{iPTH}$, ALP, BAP, $\beta$-CTX, OC, TRAcP, procollagen-1 N-terminal peptide (P1NP), and 25-dibydroxyvitamin D (25(OH)D).
Serum Ca, P, and ALP were detected using a AU5800series analyzer (Beckman Coulter, Brea, CA, USA). Serum iPTH was detected by chemiluminescence (DXC800; Beckman). Serum turnover markers TRAcP, $\beta$-CTX, BAP, and OC were detected by enzyme-linked immunosorbent assay (ELISA) and P1NP by electrochemiluminescence kit (Roche Diagnostics, Indianapolis, IN, USA). Blood samples were collected within 24 hours after surgery to retest the above variables. Laboratory parameters were measured in the Clinical Laboratory of Beijing China-Japan Friendship Hospital in China. Laboratory data were extracted from the electronic laboratory database.

\section{Statistical analysis}

All statistical analyses were performed using SPSS 22.0 version (SPSS Inc., Chicago, IL, USA). Continuous variables are displayed as means \pm standard deviations (SDs) or medians (interquartile ranges) and categorical variables as numbers (percentages). Student's $t$-test was used to compare continuous variables and Chi-square test was used for categorical variables. Paired $t$-test was used to compare postoperative and preoperative data. Covariates in the univariate analysis that reached statistical significance were chosen for further multivariate logistic regression analysis. Receiver operating characteristic (ROC) curve analysis was performed to evaluate the diagnostic value of the selected variates. A $P$ value $<0.05$ was considered statistically significant.

\section{Results}

\section{Baseline characteristics of the patients}

A total of 131 dialysis patients with SHPT who were treated with near-tPTX without AT were enrolled in this study, including $70(53.4 \%)$ males and 61 (46.6\%) females. The mean age was $47.3 \pm 11.9$ years. Among the 131 patients, 73 (55.7\%) had postoperative hypocalcemia and 43 (32.8\%) had postoperative $\mathrm{SH}$.

\section{Bone formation and resorption parameters}

Comparison of the bone formation markers (OC, ALP, $\mathrm{BAP}$, and $\mathrm{P} 1 \mathrm{NP})$ and resorption markers $[\beta-\mathrm{CTX}$ and tartrate-resistant acid phosphatase 5b (TRACb)] in SHPT patients pre- and post-operation showed that the level of iPTH decreased from $1,889.26 \pm 85.19$ to $81.5 \pm 16.76 \mathrm{pg} / \mathrm{mL}$ 
Table 1 Comparison of bone formation and resorption parameters in SHPT patients pre- and post-operation

\begin{tabular}{lcccc}
\hline Variable & Preoperation & Post-operation & t value & P value \\
\hline iPTH $(\mathrm{pg} / \mathrm{mL})$ & $1,889.26 \pm 85.19$ & $81.51 \pm 16.76$ & 21.176 & $0.000^{*}$ \\
ALP $(\mathrm{U} / \mathrm{L})$ & $465.12 \pm 44.17$ & $435.72 \pm 41.01$ & 1.407 & 0.162 \\
OC & $55.10 \pm 1.50$ & $59.19 \pm 1.20$ & -4.045 & $0.000^{*}$ \\
BAP & $70.94 \pm 24.67$ & $70.12 \pm 24.35$ & 1.367 & 0.175 \\
P1NP & $980.71 \pm 323.54$ & $949.34 \pm 339.15$ & 1.858 & 0.066 \\
$\beta$-CTX & $6.95 \pm 0.52$ & $2.04 \pm 0.14$ & 10.793 & $0.000^{*}$ \\
TRACb & $15.32 \pm 1.93$ & $9.81 \pm 0.82$ & 3.142 & $0.002^{*}$ \\
\hline
\end{tabular}

*, $\mathrm{P}<0.05$. iPTH, intact parathyroid hormone; ALP, alkaline phosphatase; OC, osteocalcin; BAP, bone alkaline phosphatase; PINP, procollagen type $1 \mathrm{~N}$-terminal propeptide; $\beta$-CTX, $\beta$-cross-linked C-telopeptide of type 1 collagen; TRACb, tartrate-resistant acid phosphatase $5 \mathrm{~b}$.

after operation $(\mathrm{P}<0.05)$. $\beta$-CTX and TRACb significantly decreased but OC increased after near-tPTX $(\mathrm{P}<0.05)$. The other three osteoblast markers (ALP, BAP, and PINP) showed no significant difference before and after near-tPTX $(\mathrm{P}>0.05)$ (Table 1). These results indicated that the activity of osteoclasts was reduced, while the osteoblasts were not affected.

\section{Comparison of parameters between patients with and without postoperative $S H$}

The baseline characteristics, presenting symptoms, and preoperative laboratory parameters of all patients are shown in Table 2. We divided patients into an SH group and nonSH group based on their postoperative serum Ca level ( $\mathrm{Ca}<1.875 \mathrm{mmol} / \mathrm{L}$ and $\mathrm{Ca}<2.10 \mathrm{mmol} / \mathrm{L}$, respectively). Comparison between the two groups showed significant differences in preoperative serum iPTH, ALP, Ca, BAP, and OC $(\mathrm{P}<0.05)$. In the multivariate logistic regression model, preoperative serum ALP was an independent predictor of postoperative $\mathrm{SH}(\mathrm{P}<0.05)$ (Table 3).

\section{ROC curves for preoperative serum ALP}

Preoperative serum ALP was found to be an independent predictor of postoperative SH. We used ROC curves to assess the potential application of plasma metabolite signatures as biomarkers for the pre-diagnosis of postoperative $\mathrm{SH}$. Based on the ROC curve, the critical value of ALP was $277 \mathrm{U} / \mathrm{L}$. The sensitivity of preoperative serum ALP was 73.8\% [95\% confidence interval (CI): 0.589 to 0.847 ] and the specificity was $63.2 \%$ (95\% CI:

\subsection{7 to 0.726$)$ (Figure 1 ).}

\section{Discussion}

PTX is an efficient intervention for CKD patients with SHPT, especially for refractory SHPT, present guidelines don't support one surgical approach of PTX over the other, so the surgeon has the right to make the choice (1). Several studies have shown a clinically beneficial effect of PTX on cardiovascular and all-cause mortality in dialysis patients with SHPT (20). However, the development of postoperative hypocalcemia is a common complication after PTX and is reported to occur in $20-85 \%$ of the patients (15). Tsai et al. reported that more than one-third of dialysis patients experienced SH following PTX (22). Compared with primary HPT, PTX may cause more cases of hypocalcemia among ESRD patients with SHPT (23). In this study, the incidence of postoperative hypocalcemia was $55.7 \%(73 / 131)$ and $\mathrm{SH}$ was $32.8 \%$ (43/131), which was consistent with previous studies (22). Moreover, postoperative hypocalcemia was mainly caused by postoperative $\mathrm{SH}$.

SHPT is characterized by high transport osteopathy. It is estimated that about $60 \%$ of maintenance dialysis (MHD) patients show high transforming renal osteodystrophy (24). When iPTH is higher than $450 \mathrm{pg} / \mathrm{mL}$ (25), high transport osteodystrophy is expected to occur in all patients undergoing regular dialysis. The iPTH of SHPT patients receiving PTX is usually $>800 \mathrm{pg} / \mathrm{mL}$, and osteoblasts and osteoclasts are upregulated in coupling (25). This leads to renal osteodystrophy due to high turnover. However, PTX can induce a decline in prolonged elevation of serum iPTH 
Table 2 Comparison of demographic and laboratory data between patients with and without postoperative SH $(\mathrm{Ca}<1.875 \mathrm{mmol} / \mathrm{L})$

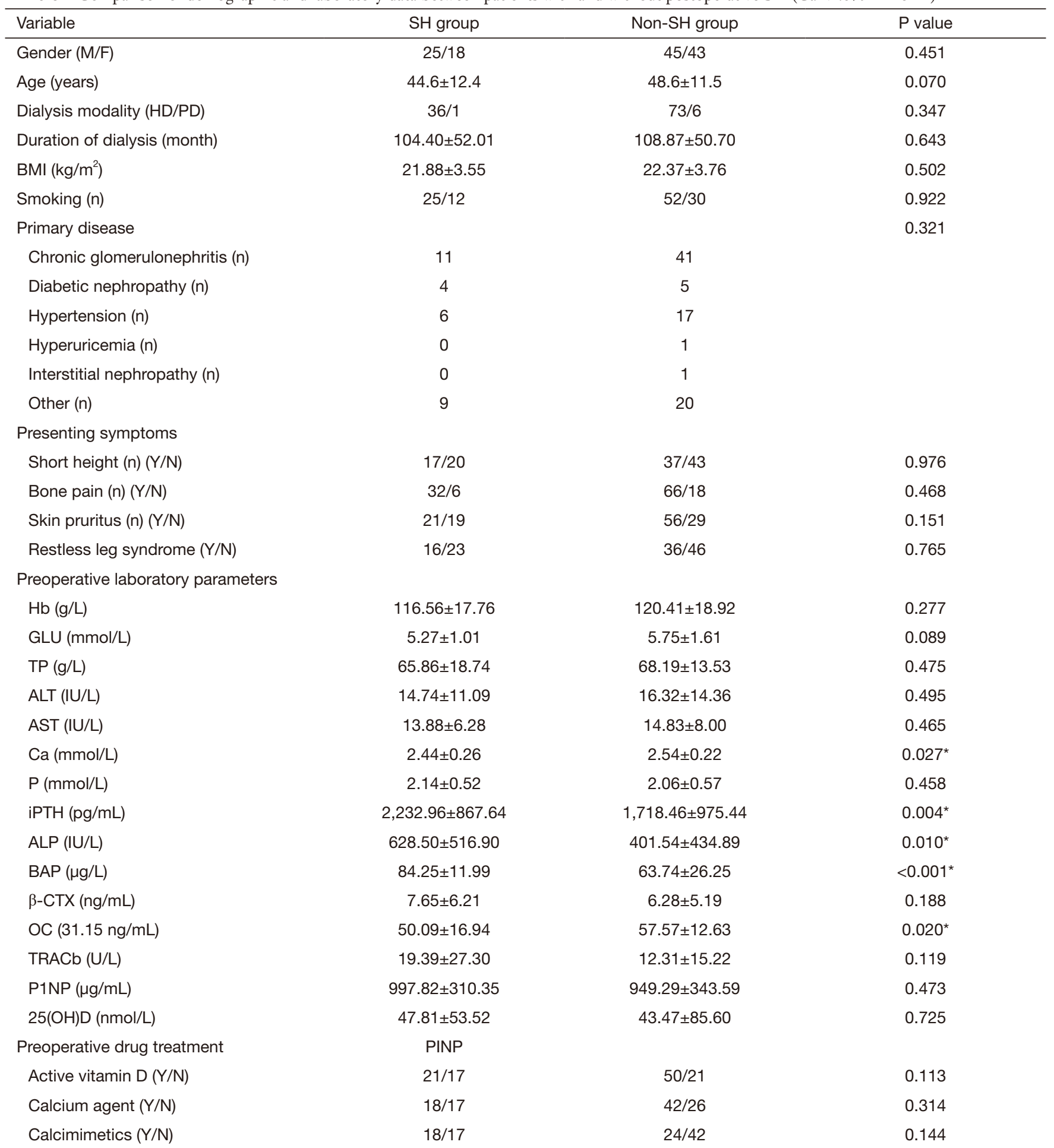

*, $\mathrm{P}<0.05 . \mathrm{SH}$, postoperative patients with severe hypocalcemia; non-SH, postoperative patients without severe hypocalcemia; HD, hemodialysis; PD, peritoneal; BMI, body mass index; Hb, hemoglobin; GLU, blood glucose; TP, total protein; ALT, alanine transaminase; AST, aspartate aminotransferase; Ca, serum calcium; P, serum phosphorus; iPTH, intact parathyroid hormone; ALP, alkaline phosphatase; BAP, bone-specific alkaline phosphatase; $\beta$-CTX, $\beta$-cross-linked C-telopeptide of type 1 collagen; OC, osteocalcin; TRACb, tartrateresistant acid phosphatase 5b; PINP, procollagen type $1 \mathrm{~N}$-terminal propeptide; 25(OH)D, 25-dihydroxyvitamin D; Y, yes; N, no. 
Table 3 Multivariate logistic regression analysis for the development of SH $(\mathrm{Ca}<1.875 \mathrm{mmol} / \mathrm{L})$ after parathyroidectomy

\begin{tabular}{lccc}
\hline Variable & OR & $95 \% \mathrm{Cl}$ & P value \\
\hline PreALP $(\mathrm{IU} / \mathrm{L})$ & 0.15 & $0.04-0.48$ & $0.002^{*}$ \\
PreiPTH $(\mathrm{pg} / \mathrm{mL})$ & 1.0 & $0.99-1.00$ & 0.397 \\
Active vitamin D $(\mathrm{Y})$ & 0.35 & $0.11-1.15$ & 0.084
\end{tabular}

*, $\mathrm{P}<0.05$. iPTH, intact parathyroid hormone; ALP, alkaline phosphatase; OR, odds ratio; $\mathrm{Cl}$, confidence interval, $\mathrm{Y}$, yes.

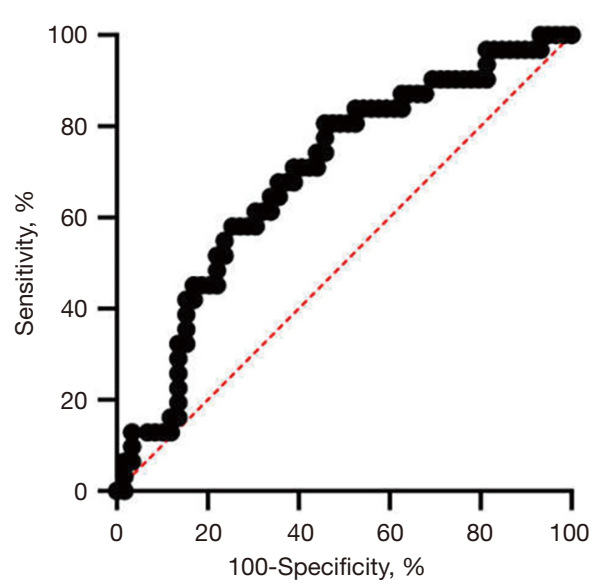

Figure 1 ROC curve for preoperative serum ALP. ROC, receiver operating characteristic; ALP, alkaline phosphatase.

level, which inhibits osteoclast activity and bone resorption. However, osteoblast activity and new bone formation continue, leading to an influx of calcium into bone tissue with an obvious increase in bone remineralization, resulting in hypocalcemia after PTX $(22,25)$. In the present study, prior to surgery, bone metabolism-regulating hormone iPTH, bone formation markers (ALP, BAP, OC, and PINP), and bone absorption markers ( $\beta$-CTX and TRACb) were significantly higher than normal levels, indicating that osteoclasts and osteoblasts were upregulated, which is characteristic of high transport osteodystrophy. After neartPTX, iPTH decreased significantly within a short period, and bone formation marker OC was significantly higher than before surgery $(\mathrm{P}<0.05)$. The other 3 bone formation markers, ALP, BAP, and PINP, did not show significant difference before and after n-tPTX. In contrast, bone absorption markers TRACb and $\beta$-CTX were significantly lower postsurgery $(\mathrm{P}<0.05)$, indicating that the activity of osteoblasts and new bone formation continued or increased in the early stage after PTX, while the activity of osteoclasts and bone resorption decreased. This explains the high incidence of postoperative hypocalcemia $(55.7 \%)$ and $\mathrm{SH}$ $(32.8 \%)$ after near-tPTX in our study $(22,24,25)$. SH can cause tetany, seizures, cardiac arrhythmias, possible sudden death, and other serious consequences (22). Therefore, early prediction and identification of postoperative $\mathrm{SH}$ is important. The relevant risk factors for postoperative $\mathrm{SH}$ include gender, age, body weight, size of resected parathyroid glands, preoperative serum iPTH level, preoperative serum ALP level, serum urea nitrogen concentration, preoperative hyperphosphatemia, and preoperative hypocalcemia (18). In the present study, preoperative serum ALP was an independent risk factor for predicting postoperative $\mathrm{SH}$, with patients possessing a preoperative serum ALP $>277$ U/L susceptible to postoperative $\mathrm{SH}$. Hence, patients with preoperative serum ALP > 277 U/L should be carefully monitored for the occurrence of SH after PTX, and a higher dose of calcium could be given post-surgery to avoid SH caused by PTX.

ALP is widely distributed in all organs of the human body and comprises several different types of isoenzymes. In adults, liver and bone isoenzymes constitute the major part of the serum ALP pool, while intestinal isoenzyme is found in small amounts ( $<20 \%$ of total ALP) in a minority of patients (about 20\%) (26). Elevated ALP occurs in both liver disease and bone disease. Serum ALP levels can reflect bone-specific ALP levels in patients with normal liver function (27). A measure of total ALP (tALP) has been recommended as an auxiliary method for diagnosing and evaluating CKD-MBD. If the level is high, further examination of liver function is needed to exclude the effect of liver disease on tALP, which is an indicator of the activity of osteoblasts. Loke et al. reported that preoperative serum ALP and postoperative hypocalcemia had a strong correlation in 29 patients with primary HPT undergoing PTX. Patients were not susceptible to symptomatic postoperative hypocalcemia when ALP $<340$ U/L (26). Yang et al. reported that preoperative ALP was associated with postoperative hypocalcemia but did not provide an ALP reference threshold for postoperative hypocalcemia (17). Therefore, this study investigated the independent risk factors of postoperative $\mathrm{SH}$ in dialysis patients with SHPT after near-tPTX and found that preoperative serum ALP $>277 \mathrm{U} / \mathrm{L}$ was a predictor of postoperative hypocalcemia. For these patients, we should measure their serum calcium concentration two to four times/day. Meanwhile intravenous calcium (1 to $2 \mathrm{~g}$ of calcium gluconate in $50 \mathrm{~mL}$ of 5 percent dextrose), oral calcium (2-4 g of elemental calcium/day) with Vitamin D 
and high-calcium bath $(1.75 \mathrm{mmol} / \mathrm{L})$ can be used in these patients $(28,29)$. However, a limitation of our study was the small sample size.

In summary, the results showed that the incidence of postoperative hypocalcemia was $55.7 \%(73 / 131)$ and $\mathrm{SH}$ was $32.8 \%$ (43/131) in dialysis patients with SHPT following near-tPTX. Preoperative serum ALP was an independent predictor for postoperative SH. Dialysis SHPT patients with preoperative serum ALP >277 U/L were susceptible to postoperative SH. This preoperative difference can help physicians to identify high-risk $\mathrm{SH}$ patients.

\section{Acknowledgments}

Funding: This work was supported by Beijing Municipal Science \& Technology Commission (Z191100006619014).

\section{Footnote}

Reporting Checklist: The authors have completed the STARD reporting checklist. Available at https://dx.doi. org/10.21037/apm-21-2509

Data Sharing Statement: Available at https://dx.doi. org/10.21037/apm-21-2509

Conflicts of Interest: All authors have completed the ICMJE uniform disclosure form (available at https://dx.doi. org/10.21037/apm-21-2509). The authors have no conflicts of interest to declare.

Ethical Statement: The authors are accountable for all aspects of the work in ensuring that questions related to the accuracy or integrity of any part of the work are appropriately investigated and resolved. This retrospective cohort study was approved by the ethics committee of China-Japan Friendship Hospital (No.:2019-SDZL-12). Given the retrospective nature of the study, formal informed consent was exempted by our institutional board. The study was conducted in accordance with the guidelines of the Declaration of Helsinki (as revised in 2013).

Open Access Statement: This is an Open Access article distributed in accordance with the Creative Commons Attribution-NonCommercial-NoDerivs 4.0 International License (CC BY-NC-ND 4.0), which permits the noncommercial replication and distribution of the article with the strict proviso that no changes or edits are made and the original work is properly cited (including links to both the formal publication through the relevant DOI and the license). See: https://creativecommons.org/licenses/by-nc-nd/4.0/.

\section{References}

1. Johal M, Levin A. Vitamin D and parathyroid hormone in general populations: understandings in 2009 and applications to chronic kidney disease. Clin J Am Soc Nephrol 2009;4:1508-14.

2. Kalantar-Zadeh K, Shah A, Duong U, et al. Kidney bone disease and mortality in CKD: revisiting the role of vitamin $\mathrm{D}$, calcimimetics, alkaline phosphatase, and minerals. Kidney Int Suppl 2010;(117):S10-21.

3. Horl $\mathrm{WH}$. The clinical consequences of secondary hyperparathyroidism: focus on clinical outcomes. Nephrol Dial Transplant 2004;19:v2-v8.

4. Jiang $\mathrm{H}, \mathrm{Xu} \mathrm{ZH}$, Zhang $\mathrm{L}$, et al. Investigation of mineral and bone metabolic disorders in pre-dialysis patients with chronic kidney disease at stage 3 to 5 . Chi J Blood Purif 2012;11:360-4.

5. Jofré R, López Gómez JM, Menárguez J, et al. Parathyroidectomy: whom and when? Kidney Int Suppl 2003;(85):S97-100.

6. Mizobuchi M, Ogata H, Koiwa F. Secondary Hyperparathyroidism: Pathogenesis and Latest Treatment. Ther Apher Dial 2019;23:309-18.

7. Parfrey PS, Chertow GM, Block GA, et al. The clinical course of treated hyperparathyroidism among patients receiving hemodialysis and the effect of cinacalcet: the EVOLVE trial. J Clin Endocrinol Metab 2013;98:4834-44.

8. Block GA, Klassen PS, Lazarus JM, et al. Mineral metabolism, mortality, and morbidity in maintenance hemodialysis. J Am Soc Nephrol 2004;15:2208-18.

9. Slinin Y, Foley RN, Collins AJ. Calcium, phosphorus, parathyroid hormone, and cardiovascular disease in hemodialysis patients: the USRDS waves 1,3 , and 4 study. J Am Soc Nephrol 2005;16:1788-93.

10. Goldfarb M, Gondek SS, Lim SM, et al. Postoperative hungry bone syndrome in patients with secondary hyperparathyroidism of renal origin. World J Surg 2012;36:1314-9.

11. Isakova T, Nickolas TL, Denburg M, et al. KDOQI US Commentary on the 2017 KDIGO Clinical Practice Guideline Update for the Diagnosis, Evaluation, Prevention, and Treatment of Chronic Kidney DiseaseMineral and Bone Disorder (CKD-MBD). Am J Kidney Dis 2017;70:737-51. 
12. Schneider R, Slater EP, Karakas E, et al. Initial parathyroid surgery in 606 patients with renal hyperparathyroidism. World J Surg 2012;36:318-26.

13. Shen Q, Xiang W, Ye S, et al. Plasma metabolite biomarkers related to secondary hyperparathyroidism and parathyroid hormone. J Cell Biochem 2019;120:15766-75.

14. Chen L, Wang K, Yu S, et al. Long-term mortality after parathyroidectomy among chronic kidney disease patients with secondary hyperparathyroidism: a systematic review and meta-analysis. Ren Fail 2016;38:1050-8.

15. Rudser KD, de Boer IH, Dooley A, et al. Fracture risk after parathyroidectomy among chronic hemodialysis patients. J Am Soc Nephrol 2007;18:2401-7.

16. Cheng SP, Lee JJ, Liu TP, et al. Parathyroidectomy improves symptomatology and quality of life in patients with secondary hyperparathyroidism. Surgery 2014;155:320-8.

17. Yang M, Zhang L, Huang L, et al. Factors predictive of critical value of hypocalcemia after total parathyroidectomy without autotransplantation in patients with secondary hyperparathyroidism. Ren Fail 2016;38:1224-7.

18. Ho LY, Wong PN, Sin HK, et al. Risk factors and clinical course of hungry bone syndrome after total parathyroidectomy in dialysis patients with secondary hyperparathyroidism. BMC Nephrol 2017;18:12.

19. Sun X, Zhang X, Lu Y, et al. Risk factors for severe hypocalcemia after parathyroidectomy in dialysis patients with secondary hyperparathyroidism. Sci Rep 2018;8:7743.

20. Apetrii M, Goldsmith D, Nistor I, et al. Impact of surgical parathyroidectomy on chronic kidney disease-mineral and bone disorder (CKD-MBD) - A systematic review and meta-analysis. PLoS One 2017;12:e0187025.

21. Graciolli FG, Neves KR, Barreto F, et al. The complexity of chronic kidney disease-mineral and bone disorder across stages of chronic kidney disease. Kidney Int 2017;91:1436-46.

22. Tsai WC, Peng YS, Chiu YL, et al. Risk factors for severe hypocalcemia after parathyroidectomy in prevalent dialysis patients with secondary hyperparathyroidism. Int Urol Nephrol 2015;47:1203-7.

23. Mittendorf EA, Merlino JI, McHenry CR. Postparathyroidectomy hypocalcemia: incidence, risk factors, and management. Am Surg 2004;70:114-9; discussion 119-20.

24. Covic A, Vervloet M, Massy ZA, et al. Bone and mineral disorders in chronic kidney disease: implications for cardiovascular health and ageing in the general population. Lancet Diabetes Endocrinol 2018;6:319-31.

25. Yang G, Ge Y, Zha X, et al. Peritoneal dialysis can alleviate the clinical course of hungry bone syndrome after parathyroidectomy in dialysis patients with secondary hyperparathyroidism. Int Urol Nephrol 2019;51:535-42.

26. Loke SC, Tan AW, Dalan R, et al. Pre-operative serum alkaline phosphatase as a predictor for hypocalcemia postparathyroid adenectomy. Int J Med Sci 2012;9:611-6.

27. Bauer D, Krege J, Lane N, et al. National Bone Health Alliance Bone Turnover Marker Project: current practices and the need for US harmonization, standardization, and common reference ranges. Osteoporos Int 2012;23:2425-33.

28. Tohme JF, Bilezikian JP. Diagnosis and treatment of hypocalcemic emergencies. The Endocrinologist 1996; 6:10.

29. Clair F, Leenhardt L, Bourdeau A, et al. Effect of calcitriol in the control of plasma calcium after parathyroidectomy. A placebo-controlled, double-blind study in chronic hemodialysis patients. Nephron 1987;46:18-22.

(English Language Editor: A. Muijlwijk)
Cite this article as: Zou Y, Zhang N, Tang Y, Zhan Z, Yang M, Lu Y, Li GS, Zhang L. Predictive markers for severe hypocalcemia in dialysis patients with secondary hyperparathyroidism after near-total parathyroidectomy. Ann Palliat Med 2021;10(10):10712-10719. doi: 10.21037/apm-21-2509 